\title{
Genetic heterogeneity of Usher syndrome type II: localisation to chromosome $5 \mathrm{q}$
}

\author{
S Pieke-Dahl, C G Möller, P M Kelley, L M Astuto, C W R J Cremers, M B Gorin, \\ W J Kimberling
}

Genetics Department, Boys Town National

Research Hospital, 555 N 30th Street, Omaha, NE 68131 USA

S Pieke-Dahl

P M Kelley

L M Astuto

W J Kimberling

Department of Audiology,

Sahlgrenska University

Hospital, Götebörg,

Sweden

C G Möller

Department of

Oto-Rhino-Laryngology, The Usher syndromes are a group of autoUniversity Hospital, Nijmegan, The

Netherlands

C W R J Cremers

Department of Ophthalmology, University of

Pittsburgh School of Medicine, Pittsburgh, PA, USA

M B Gorin

Correspondence to: Dr Kimberling,

kimber@boystown.org

Revised version received 10 September 1999 Accepted for publication 1 December 1999

(F Med Genet 2000;37:256-262)

Keywords: Usher syndrome; chromosome 5q; retinitis pigmentosa; hearing loss somal recessive disorders characterised by retinitis pigmentosa (RP) with sensorineural hearing loss and variable vestibular areflexia. There is extensive clinical and genetic heterogeneity in Usher syndrome. The distinguishing characteristics of Usher type I are profound hearing loss and vestibular dysfunction. Moderate to severe high frequency hearing loss and normal vestibular function are representative of Usher type II. Progressive hearing loss is typically seen in Usher type III. ${ }^{2}$ Usher III appears to be relatively common in the Finnish Usher population, but rare elsewhere ${ }^{3}$; the USH3 gene has been localised to $3 \mathrm{q} 25$. $^{4}$

The clinical picture of Usher syndrome type II is complicated because subtle variations

\begin{abstract}
Usher syndrome is a group of autosomal recessive disorders that includes retinitis pigmentosa (RP) with hearing loss. Usher syndrome type II is defined as moderate to severe hearing loss with RP. The USH $2 A$ gene at 1q41 has been isolated and characterised. In 1993, a large Usher II family affected with a mild form of RP was found to be unlinked to 1q41 markers. Subsequent linkage studies of families in our Usher series identified several type II families unlinked to $U S H 2 A$ and $U S H 3$ on $3 q 25$. After a second unlinked family with many affected members and a mild retinal phenotype was discovered, a genome search using these two large families showed another Usher II locus on 5q (two point lod $=3.1$ at D5S484). To date, we have identified nine unrelated 5q linked families (maximum combined multipoint lod $=5.86$ ) as well as three Usher II families that show no significant linkage to any known Usher loci. Haplotype analysis of $5 q$ markers indicates that the new locus is flanked by D5S428 and D5S433. Review of ophthalmological data suggests that RP symptoms are milder in $5 q$ linked families; the RP is often not diagnosed until patients near their third decade. Enamel hypoplasia and severe, very early onset RP were observed in two of the three unlinked families; dental anomalies have not been previously described as a feature of Usher type II.
\end{abstract}

within the Usher II hearing phenotype have been observed in several Dutch studies. In the first, three of 13 type II patients had a mild but definite progression of hearing loss unrelated to presbycusis; these three families showed linkage to USH $2 A$ and not to USH3. ${ }^{5}$ A subsequent cross sectional study was done with 27 patients whose status as Usher type IIa had also been confirmed by linkage analysis. When hearing threshold was compared against age, significant progression of hearing impairment was observed, but at a much slower rate than previously reported for Usher III patients. ${ }^{6}$ All putative Usher II families should be excluded from linkage to USH3 to negate the possibility of phenotypic overlap between the two subtypes. Good quality serial audiograms (when available) can be used in conjunction with linkage analysis to distinguish between Usher IIa and Usher III in some cases.

Mutations in the USH2A gene on chromosome 1q41 appear to be responsible for most cases of Usher type II. ${ }^{7}$ The gene has 21 exons and codes for usherin, a novel protein whose structure is partially homologous with the laminin protein group. The function of usherin is not yet understood but it has been postulated that it is a cell adhesion molecule or forms part of the basement membrane. The genomic structure of $U S H 2 A$ has been determined and several new USH $2 A$ mutations have recently been found. ${ }^{8}$

Genetic heterogeneity within the Usher II subtype was first noticed when a family with an Usher II phenotype (family 735) was found to be unlinked to $1 \mathrm{q} 41$ markers flanking the USH $2 A$ locus. ${ }^{9}$ A subsequent heterogeneity study of 29 Usher II Dutch families identified three that were unlinked to the USH2A region. ${ }^{10}$ There was no evidence of significant linkage to 3q25 (USH3) in this specific sample. A preliminary study of the proportion of different linkages in a sample of pooled Usher type II and type III families showed that $82 \%$ showed linkage to USH $2 A$ and $12 \%$ showed linkage to USH 3 , but $3.4 \%$ were unlinked to both $1 \mathrm{q}$ and 3q. ${ }^{11}$

The question remained as to exactly where this new Usher II gene was located in the human genome. The previous studies had identified several unlinked Usher II families, but these were too small and uninformative for a productive genome search. Then, an unlinked family (1848) with multiple affected sibs and a retinal phenotype almost identical to that of family 735 was discovered. These two highly informative families were chosen for the genome search. 
Table 1 Two point lod scores for $5 q$ markers with 12 families $(\theta=0.0-0.3)$ and results of $A$ test for heterogeneity ( $\alpha$ and $\chi^{2}$ ). Lods for D5S2029 are higher because unlinked families 49,1322 , and 843 were not typed for this marker

\begin{tabular}{lcllllll}
\hline Locus & 0.0 & 0.05 & 0.1 & 0.2 & 0.3 & $a$ & $\chi_{(1)}^{2}$ \\
\hline D5S2029 & 4.487 & 4.023 & 3.543 & 2.525 & 1.454 & 1 & 0.0 \\
D5S617 & -5.49 & 1.238 & 1.313 & 0.957 & 0.521 & 1 & 0.0 \\
D5S484 & -7.438 & 4.491 & 4.217 & 3.062 & 1.723 & 1 & 0.0 \\
D5S495 & -8.922 & 1.369 & 1.882 & 1.718 & 1.025 & 0.68 & 6.8 \\
D5S409 & -6.191 & 2.593 & 2.574 & 1.933 & 1.106 & 1 & 0.0 \\
D5S505 & -10.627 & 3.656 & 3.923 & 3.176 & 1.904 & 0.72 & 8.0 \\
D5S485 & 0.487 & 2.785 & 2.663 & 2.047 & 1.23 & 0.96 & 0.01 \\
\hline
\end{tabular}

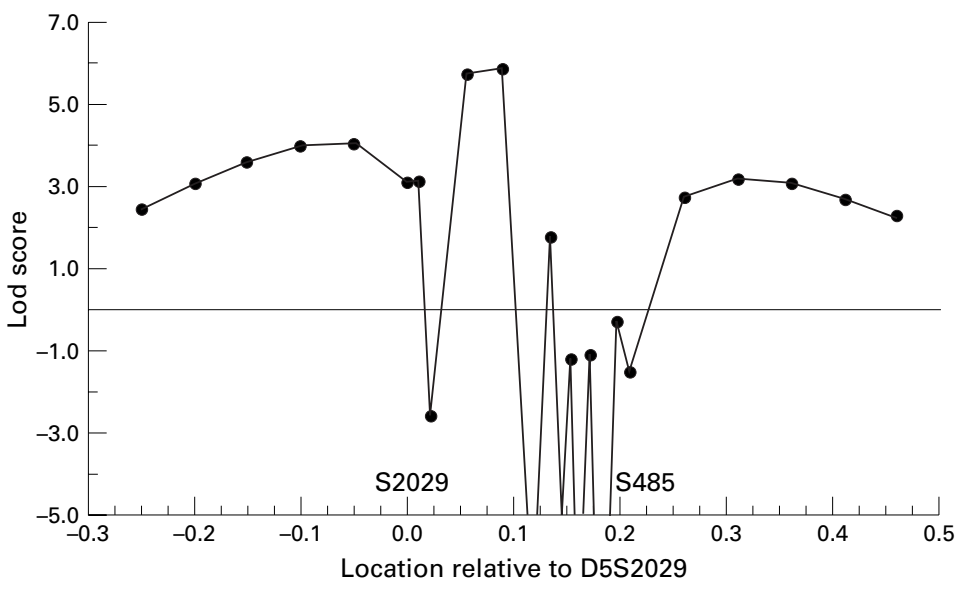

Figure 1 Multipoint lod score for $5 q$ markers versus Usher II.

\section{Methods}

CLINICAL STUDIES

Families with clinical diagnoses of Usher II were ascertained through physician's referral and self-referral. The specific audiometric, vestibular, and ophthalmic criteria used for a clinical diagnosis of Usher type I and type II have been previously described. ${ }^{12}$ The average Usher II patient will present with (1) RP, symptoms include nyctalopia, an extinguished or subnormal electroretinogram (ERG), and restricted visual fields; (2) congenital, relatively stable, "sloping" hearing loss, moderate in the low frequencies and severe in the higher frequencies; (3) intact vestibular responses; and (4) autosomal recessive inheritance pattern.

DNA AND PCR MARKERS

Blood samples were collected and DNA was extracted from lymphocytes using the PureGene DNA extraction kit (Gentra Systems Inc) according to the vendor's instructions. ABI Prism marker panels (Version 1, Perkin Elmer Inc) that spanned the entire genome at

Table 2 Clinical summary of 12 Usher II families

\begin{tabular}{lllllll}
\hline Family & Origin & HOMOG & Audiogram & $\begin{array}{l}\text { RP DX } \\
(y)\end{array}$ & $\begin{array}{l}\text { RP } \\
\text { symptoms }\end{array}$ & Enamel \\
\hline 735 & English/USA & 0.9969 & Moderate-severe & 30 & Mild & - \\
1848 & Irish/USA & 0.9945 & Moderate-severe & 32 & Mild & Normal \\
964 & Swedish & 0.9837 & Moderate-severe & 31 & Mild & Normal \\
1121 & German/USA & 0.9187 & Severe-profound & 28 & Mild-mod & Normal \\
1417 & Dutch & 0.9187 & Moderate-severe & 26 & Moderate & Normal \\
2488 & USA & 0.8776 & Severe-profound & 32 & Mild & Normal \\
1389 & Dutch & 0.7907 & Moderate-severe & 6 & Moderate & Normal \\
736 & English/USA & 0.7779 & Moderate-severe & 30 & Moderate & - \\
1372 & Dutch & 0.7392 & Moderate-severe & 15 & Moderate & Normal \\
843 & English/USA & 0.7378 & Severe-profound & 5 & Severe & Hypoplasia \\
49 & Dutch/USA & 0.0 & Severe-profound & 3 & Severe & Hypoplasia \\
1322 & USA & 0.0 & Severe-profound & 17 & Moderate & - \\
\hline
\end{tabular}

$(-)$ denotes data not currently available. approximately $10 \mathrm{cM}$ intervals were purchased for the genome search. Additional PCR markers were later chosen based on chromosomal position, heterozygosity index, and fragment size for the purpose of linkage mapping. PCR primer sequences were obtained from (1) published linkage maps, (2) Whitehead Institute Center for Genome Research (WI-CGR) Human Physical Mapping Project database (http://www.genome.wi.mit.edu), and (3) the Genome Data Base (http://www.gdb.org). All blue, green, and yellow fluorescent dye labelled oligonucleotide primer pairs were constructed by Integrated Technologies Inc.

GENOTYPING

Genotyping was done using AmpliTaq Gold DNA polymerase in multiplexed PCRs with an 877 Integrated ThermalCycler robotic system (Perkin Elmer Inc) according to standardised protocol. Pooled reactions were loaded onto a $6 \%$ denaturing gel and electrophoresed on an ABI 377A automated DNA genotyper/ sequencer. Gel lane data were generated with the GENESCAN 672 software program. The gel file data were then imported into GENOTYPER 2.0 for identification of individual marker alleles depicted as electrophoretograms.

LINKAGE ANALYSIS

Twelve Usher type II families informative for linkage were considered for this study after none showed linkage to $1 \mathrm{q} 41$ markers D1S229, D1S237, D1S490, and D1S474. Linkage to 3q25 was tested with markers D3S1299, D3S1555, D3S1280, and D3S1279. Atypical Usher I was ruled out by testing linkage with flanking markers for all six Usher I loci (Usher marker panel listed in Hereditary Hearing Loss Homepage http://hgins.uia.ac.be/dnalab/hhh). Markers D3S1578 and D3S3647 were chosen to test linkage to $3 \mathrm{p} 23-\mathrm{p} 24.2 .^{13}$ No evidence of linkage was observed for $3 p$ or any other previously known Usher loci. After the genome search detected linkage to $5 \mathrm{q}$, the new localisation was studied further by genotyping all 12 families with the following seven markers shown in map order: D5S2029-(2.2) D5S617 - (9.8) - D5S484 - (2.6) - D5S495 (1.5) - D5S409 - (2.3) - D5S505 - (2.5) D5S485. Distances in cM are presented in parentheses between adjacent markers. Interlocus distances were obtained from the National Center for Biotechnology Institute (NCBI) GeneMap '98 (http//www.ncbi.nlm.nih.gov/ genemap) and the WI-CGR STS database (http://carbon.wi. mit.edu).

Marker data were analysed with the LINKAGE program package, version 5.1. ${ }^{14}$ Both two point and a rolling multipoint analysis were performed. The rolling multipoint analysis was done using overlapping sets of four contiguous marker loci with the test locus (five point analysis). Multipoint lod scores are calculated at approximately $0.5 \mathrm{cM}$ intervals for each family. An A test of heterogeneity was performed on both two point and multipoint lod scores using HOMOG. ${ }^{15}$ The frequency of the 


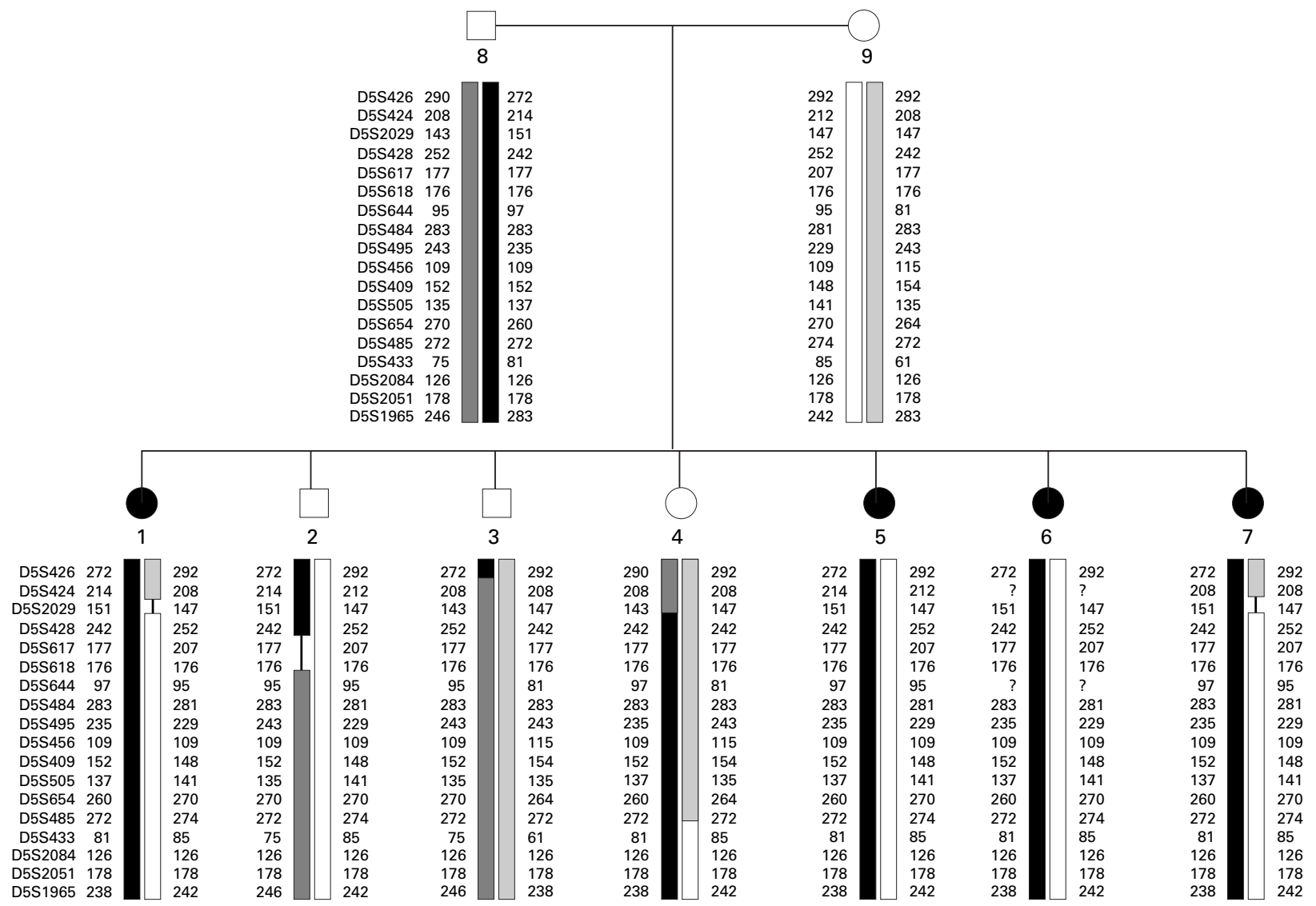

Figure 2 Family 735.

Usher II gene was arbitrarily set at 0.005 and penetrance in the mutant homozygote was assumed to be complete.

\section{Results}

A genome search was done with families 735 and 1848 using the ABI Prism 1 panels. Two point lod scores were generated for each marker until significant linkages on chromosome $5 \mathrm{q}$ were detected. The study was then expanded to include smaller unlinked families. In a preliminary report, we announced that Usher type II in 735,1848 , and two other families was linked to chromosome 5q14.3q21.3 with a combined two point lod score over 3.1 at D5S484. ${ }^{16}$

To date, 12 unlinked families have been evaluated for linkage to $5 \mathrm{q}$ with the seven markers listed in table 1. A combined two point lod score of 4.49 was observed at marker D5S484 $(\theta \max =0.05)$. Two point lod scores for each marker were then analysed by the $A$ test for heterogeneity and the results for $\alpha$ (the proportion of linked families) and $\chi^{2}$ are shown in the last two columns of table 1. The test for linkage heterogeneity $v$ homogeneity with linkage was significant for D5S495 and D5S505, with $\chi_{(1)}^{2}=6.8 \quad(\mathrm{p}<0.001)$ and $\chi_{(1)}^{2}=8.0$ $(\mathrm{p}<0.001)$, respectively.

The multipoint maximum lod score for this sample was 5.86 observed between D5S617 and D5S484 (fig 1). Multipoint lod scores between D5S495 and D5S505 were analysed by the A test for heterogeneity, yielding a significant $\chi_{(1)}^{2}=26.9 \quad(\mathrm{p}<0.001)$, establishing that both $5 \mathrm{q}$ linked and unlinked families were present in this sample. The proportion of $5 \mathrm{q}$ linked families in this sample was estimated to be 0.68 .

Conditional probabilities obtained from the HOMOG program for all 12 families are listed in table 2. Three families were highly informative and the probability of linkage to 5q markers for families 735, 1848, and 964 was estimated to be over $98 \%$ in each case. Six smaller, less informative families scored probabilities ranging from 92-74\%. Conditional probabilities of 0.0 were observed for families 49 and 1322; neither show linkage to any known Usher locus. Family 843 is small (one parent, sibship of two with one affected and one unaffected offspring), but the proband has the distinctive phenotype similar to that of family 49 .

A diagnosis of RP was first established between the ages of 28 and 32 in six of the $5 q$ linked families. Only the first diagnosed sib was considered because it was obvious that the age at diagnosis within a given family could be influenced by a positive family history. Remarkably, in family 1848, the sib diagnosed with RP at 32 years had an ERG that was diminished but not completely extinguished and the oldest sister had retained a visual field of $20^{\circ}$ by the age of 39 . An affected sib from family 964 retained $20^{\circ}$ of vision by the age of 40 as well.

In sharp contrast, affected sibs from families 49 and 843 have been afflicted with nystagmus 
since early infancy and RP symptoms were severe enough for diagnosis by ages 3 and 5, respectively. In family 49, ERGs were reduced at 2-3 years and extinguished by the age of 5 . Family 1322 exhibits a more typical Usher retinal phenotype, with diagnosis of $\mathrm{RP}$ by the age of 17 and no reported nystagmus.

These preliminary ophthalmic data indicate that $\mathrm{RP}$ in $5 \mathrm{q}$ linked families may appear as much as one decade later than typically seen in Usher syndrome and RP symptoms seem less severe. Möller et al ${ }^{1}$ reported that RP was not clinically diagnosed until around 13 years of age (range 2-18 years) in 16 Usher type I patients. RP was diagnosed at 18 years of age (range 11-28 years) in nine Usher type II patients. It is not known whether the nine Usher II patients were Usher IIa, since this study predates the subsequent localisation of $U S H 2 A$ and identification of usherin.

The degree of hearing loss in each family coincided with the extent of variation typically seen for Usher type II. ${ }^{9}$ Hearing loss ranged from moderate to severe in the lower frequencies and severe to profound in the higher frequencies in the nine $5 \mathrm{q}$ linked families. All three unlinked families had severe to profound hearing loss. Serial audiograms were not currently available for all 12 families, so the possibility of slowly progressive hearing loss unrelated to presbycusis could not be tested for the entire group. However, serial audiograms for $5 q$ linked family 1121 did provide evidence for a slowly progressive hearing loss, similar to that described for patients with USH2a. ${ }^{5}$ No significant vestibular anomalies were reported for any of the 12 families.

Surprisingly, two of three unlinked families had enamel hypoplasia, a standard clinical feature of no known Usher subtype. The teeth were described as small, brownish, or peg-like. $X$ rays showed enamel hypoplasia; the teeth tend to be "soft" and susceptible to dental caries. No dental anomalies were reported for eight of the nine $5 \mathrm{q}$ linked families.

Because ABI Prism marker panels are designed to cover an entire chromosome at an interval of approximately $10 \mathrm{cM}$ intervals, a second round of mapping with additional markers was done in an effort to reduce and refine the $5 \mathrm{q}$ critical region and to produce a linkage map of the new Usher II locus. Haplotypes of 5q markers were constructed for all 12 families. Nine were compatible with linkage to $5 q$ and two showed no linkage (843 was inconclusive). Figs 2, 3, and 4 depict family

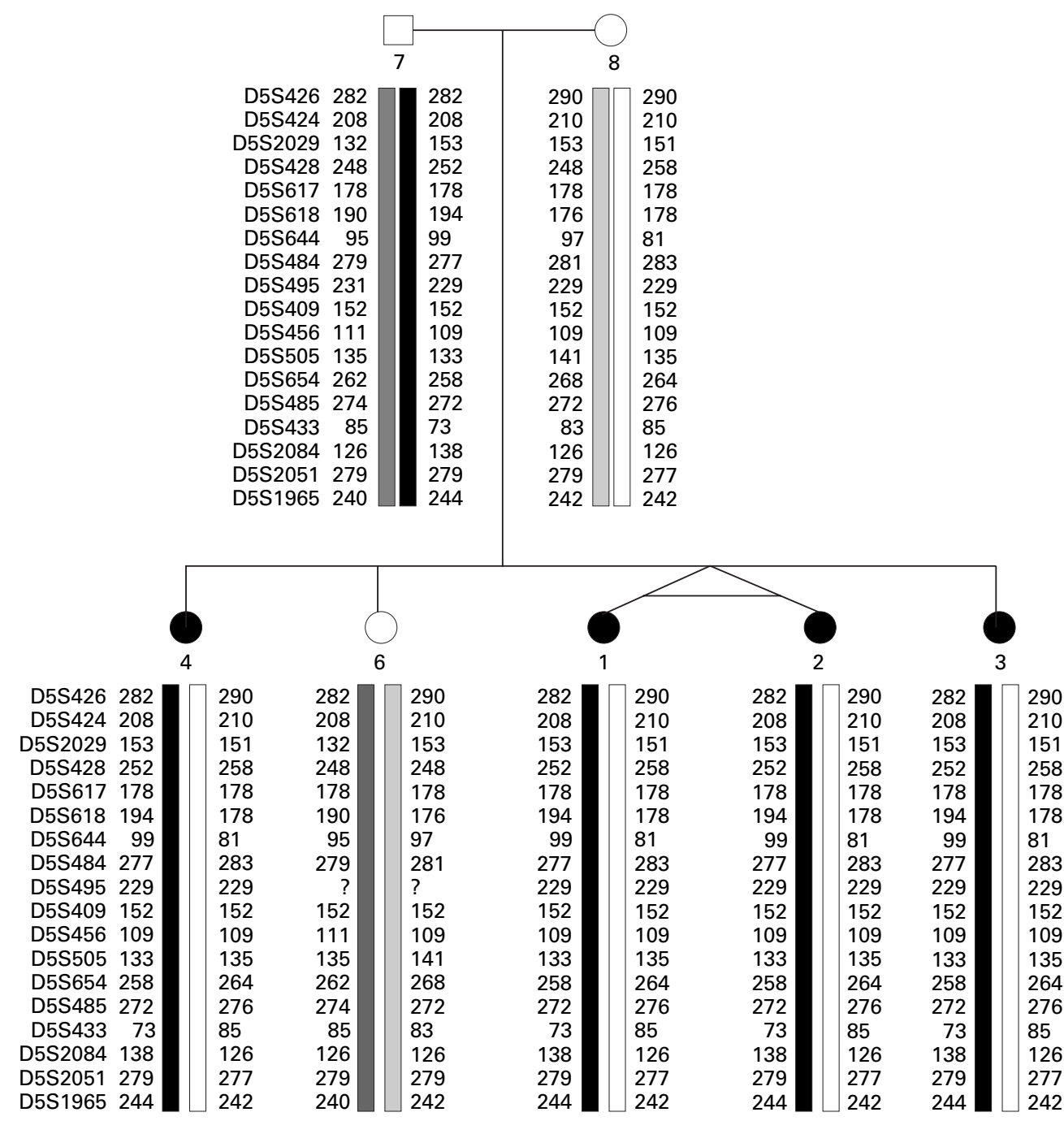

Figure 3 Family 1848. 
pedigrees for 735, 1848, and 964. Parental phases are set to reflect the minimum number of single crossovers with no double crossovers. Examination of the haplotype data shows that the Usher gene is excluded from D5S428 proximal (subject 2) and from D5S433 distal (subject 4) in family 735.

Our haplotype data reflect the gene order D5S505-D5S654-D5S485-D5S433. Although the Genome Data Base (GDB) comprehensive genetic map (http://www.gdb.org/gdb-bin/ custom) lists probable gene order as D5S505D5S433-D5S654-D5S485, localisation of the respective markers is not precise. Exclusion mapping with our families currently places D5S485 proximal to D5S433. The previously reported position of D5S433 at $112.2 \mathrm{cM}$ is relatively close to D5S485 at $112.8 \mathrm{cM} .{ }^{17}$ It is likely that future high resolution linkage mapping of the new Usher II gene will resolve the position of D5S485 relative to D5S433.

Fig 5 illustrates a composite linkage map (in $\mathrm{cM}$ ) of the 5q14-q21 region, which was constructed using data from the following sources: the recently updated NCBI Genemap'99 and the NCBI STS database (http:// www2.ncbi.nlm.nih.gov/irx), the WI-CGR STS database, GDB, the Marshfield chromosome 5 sex averaged linkage map (http:// www.marshmed.org/genetics), the Human Genome Research Center-University of
California Irvine (HGRC-UCI) chromosome 5 radiation hybrid map (http// www.chrom5.hsis.uci.edu), and our own linkage data. We estimate that the new Usher II gene lies within the chromosomal area flanked by D5S428 and D5S433 which spans approximately 16.8 cM (NCBI Genemap'99). High resolution mapping with additional $5 \mathrm{q}$ markers may expedite the identification and characterisation of this Usher II gene. The HUGO nomenclature committee has officially designated the Usher II locus on 5q14-q21 as "USH2C".

The transcription factor COUP-TFI was evaluated as a potential candidate when it was found to lie between the flanking markers that delineate the 5q locus (HGRC-UCI chromosome 5 radiation hybrid map, NCBI Genemap'99). PCR primers were designed from COUP-TFI mRNA sequence (GenBank accession number X12795). These primers were used directly to determine the intron-exon structure of the COUP-TF1 by sequencing corresponding PAC and BAC clones. Then, intronic primers were designed and used to amplify genomic DNA from affected subjects in five of the nine $5 q$ linked families. No mutations were discovered in these five families (data not shown); four have not been screened to date.

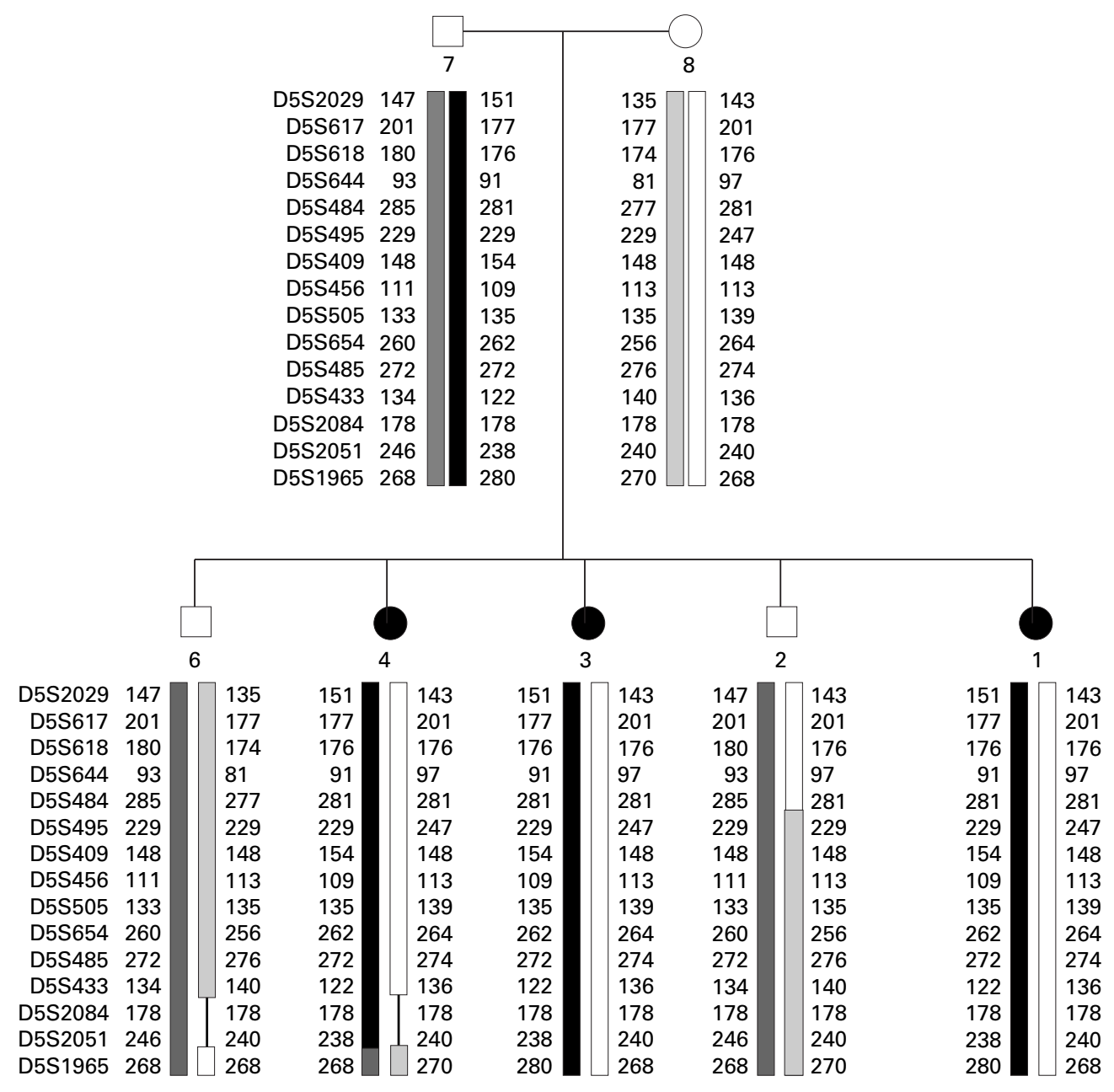

Figure 4 Family 964. 


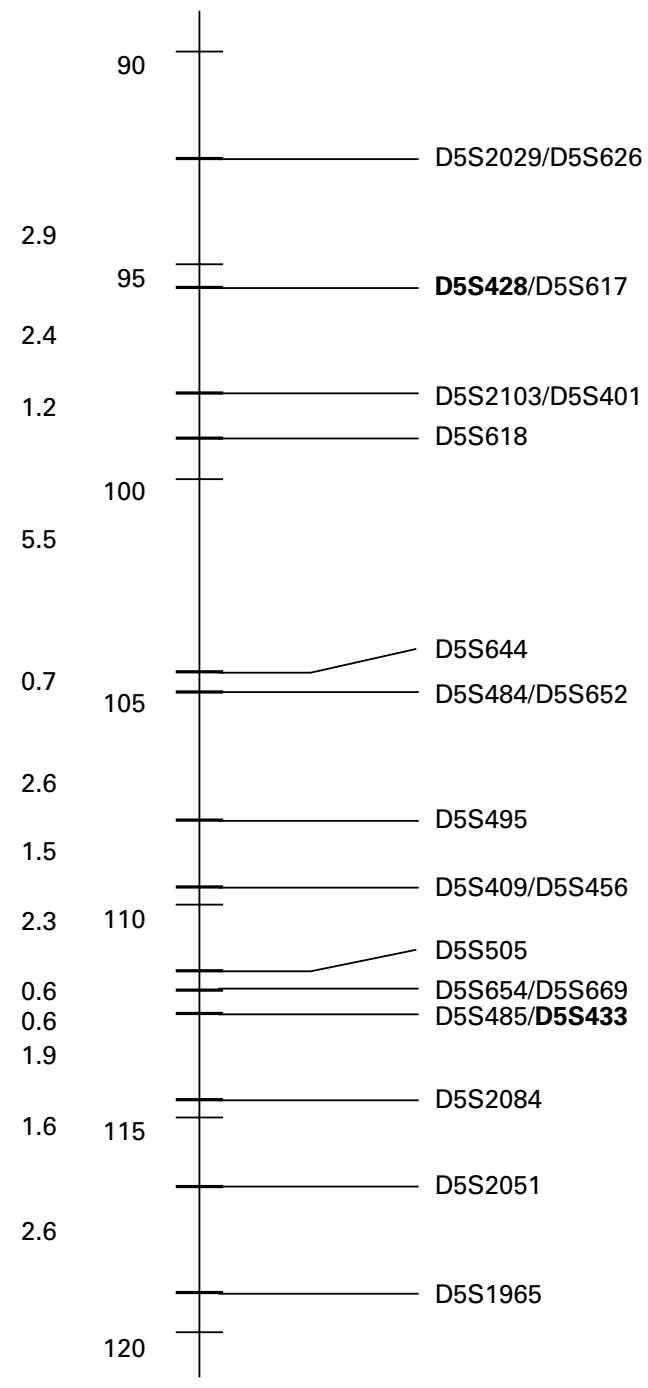

Figure 5 Linkage map of $5 q$ locus.

Discussion

A genome search using two large unlinked Usher II families with a mild RP phenotype showed another Usher II locus on 5q. A total of nine unrelated $5 \mathrm{q}$ linked families have been identified to date. Three Usher II families display no convincing evidence of linkage to any known Usher locus. The $5 \mathrm{q}$ locus is flanked by markers D5S428 and D5S433. Diagnosis of $\mathrm{RP}$ in $5 \mathrm{q}$ linked families is usually made around 30 years of age. Two of the three unlinked families have enamel hypoplasia and RP was diagnosed in early childhood. A review of singleton cases in our series of Usher families has shown several other patients with severe, early onset RP, nystagmus, and Usher II-like hearing loss. In one instance, enamel hypoplasia was discovered upon subsequent dental examination at our request.

Although enamel hypoplasia has not been previously reported as a feature of Usher syndrome specifically, Bateman et $a l^{18}$ described a 15 year old patient with RP, severe sensorineural hearing loss, and enamel hypoplasia. Radiographs showed the proband's enamel to be thinner than normal. More recently, Innis et $a l^{19}$ presented a family where three of four sibs were affected with hearing loss characteristic of Usher type II, creamywhite retinal lesions, and small, brownish molars and canines. The ERG of their 6 year old proband showed subnormal rod responses and a visual field deficit indicative of early RP. Pedigree analysis and family history indicated autosomal recessive inheritance. This clinical description resembles that of the unlinked Usher II families 49 and 843 presented here.

An atypical Usher III family with mutations in myosin VIIa (USH1B) and a mild retinal phenotype has been described by Liu et al. ${ }^{20}$ The proband presented with a severe to profound hearing loss, normal vestibular function, and RP diagnosed at 38 years. This family was unlinked to USH3, but was compatible for linkage to USH1B. Mutation analysis with DNA sequencing and SSCP analysis showed that both affected sibs had a maternal Leu651Pro substitution in exon 17 and a paternal Arg1602Gln in exon 35. In the absence of functional testing, it is difficult to be certain whether either of these missense mutations is truly pathological. Furthermore, this family was not genotyped with 1q41 markers. In light of our findings of a milder RP in $5 \mathrm{q}$ linked Usher II families, it would be interesting for this family to be genotyped for markers flanking the $5 \mathrm{q}$ Usher II locus as well.

Computer searches of the Mouse Genome Database (http://www.informatics.jax.org), the NCBI Davis mouse/human homology map (http://www.ncbi.nlm.gov/OMIM/homology), and the Human-Mouse Dysmorphology Database (http://www.hgmp.mrc.ac.uk/dhmhd) were done to predict the position of the murine version of the Usher II gene on human 5q14-q21. Mouse chromosome 13 homology group 46 listed in the NCBI Davis Homology Map appears to be the most likely homologous location.

Murine transcripts of coup-tf1 (currently known as erbal3) have been localised to various regions of the central and peripheral nervous system including the inner ear. ${ }^{21}$ erbal3 is located on the distal part of mouse chromosome 13 near $m e f 2 c,{ }^{22}$ in a region homologous to human 5q14. Although we have seen no evidence supporting COUP-TF1 as an Usher II gene so far, there are not enough data to rule out COUP-TF1's involvement as yet, and erbal3 on mouse chromosome 13 may be a murine positional candidate. COUP-TFI belongs to the steroid/thyroid hormone receptor superfamily, which modulates thyroid hormone, vitamin $\mathrm{D}$, and retinoic acid signalling pathways.

Future research in Usher syndrome type II must address both the substantial phenotypic and genetic heterogeneity of Usher II subtypes. An in depth clinical study is needed to corroborate the phenotypic differences we observed in our Usher II series. All putative Usher II families should be genotyped with $5 \mathrm{q}$ markers and examined for dental anomalies. Ascertainment of additional unlinked Usher II families with severe, early onset RP and enamel hypoplasia will increase the likelihood of a successful genome search for yet another Usher II gene. 
Our colleagues Drs Annelies van Aarem and Mariette Wagenaar generated and analysed large quantities of clinical and statistical data for detailed clinical assessments of Usher syndrome subtypes. Research associates Denise Hoover, Kate Brennan, Carol Carney, Tom Fowler, and Renee Rogers, along with research assistants Andrea Broberg and Maren Jensen, provided essential laboratory and clinical support. Administrative assistant Carole Dugan was a valuable conduit of information. We are especially grateful to colleague Michael D Weston for his insight, expertise, and persistence in the pursuit of Usher genes over the last decade. This research was approved by the BTNRH Human Subjects Oversight Committee and conform to NIH guidelines. Research was funded by grants NIHNIDCD P01 DC01813-01 NIH-R01DC00677 and grant 89-198 from the Foundation Fighting Blindness, USA.

1 Möller CG, Kimberling WJ, Davenport SL, et al. Usher syndrome: an otoneurologic study. Laryngoscope 1989;99:73-5.

2 Kimberling WJ, Möller C. Clinical and molecular genetics of Usher syndrome. F Am Acad Audiol 1995;6:63-72.

3 Karjalainen S, Pakarinen L, Terasvirta M, Kaariainen H, Vartiainen E. Progressive hearing loss in Usher's syndrome. Ann Otol Rhinol Laryngol 1989;98:863-6.

4 Sankila EM, Pakarinen L, Kaariainen H, et al. Assignment Sankila EM, Pakarinen L, Kaariainen $\mathrm{H}$, et al. Assignment
of an Usher syndrome type III (USH3) gene to of an Usher syndrome type III (USH3)
chromosome 3q. Hum Mol Genet 1995;4:93-8.

5 van Aarem A, Huygen PLM, Pinckers AJLG, et al. Stable and progressive hearing loss in type $2 \mathrm{~A}$ Usher syndrome. Ann Otol Rhinol Laryngol 1996;105:962-7.

6 Wagenaar M, van Aarem A, Huygen P, Pieke-Dahl S, Kimberling W, Cremers C. Hearing impairment related to age in Usher syndrome type 1B and 2A. Arch Otolaryngol Head Neck Surg 1999;125:441-5.

7 Eudy JD, Weston MD, Yao SF, et al. Mutation of a gene encoding a protein with extracellular matrix motifs in USH2A. Science 1998;280:1753-6.

8 Weston MD, Eudy JD, Fujita T, et al. Determination of the genomic structure of USH $2 \mathrm{~A}$, and the identification of novel mutations causing autosomal recessive Usher syndrome type IIa. Am f Hum Genet (submitted).

9 Pieke-Dahl S, Kimberling WJ, Gorin MB, et al. Genetic heterogeneity of Usher syndrome type II. $7 \mathrm{Med}$ Genet 1993;30:843-8.
10 Pieke Dahl S, van Aarem A, Dobin A, Cremers CWRJ, Kimberling WJ. Genetic heterogeneity of Usher sydrome type II in a Dutch population. F Med Genet 1996;33:753-7.

11 Pieke-Dahl S, Weston MD, Kimberling WJ. Genetic heterogeneity of US. Am F Hum Genet Suppl 1997;61: A291.

12 Smith RJH, Berlin CJ, Heimancik JF, et al. Clinical diagnosis of the Usher syndromes. Am $\mathcal{F}$ Med Genet 1994;50:32-

13 Hmani M, Ghorbel A, Boulila-Elgaied A, et al. A novel locus for Usher syndrome type II, USH2B, maps to chromosome 3 at p23-24.2. Eur f Hum Genet 1999;7:363-7.

14 Lathrop GM, Lalouel JM, Julier C, Ott J. Multilocus linkage analysis in humans: detection of linkage and estimation of recombination. Am f Hum Genet 1985;37:482-98.

15 Ott J. Analysis of human genetic linkage. Baltimore: Johns Hopkins University Press, 1991.

16 Pieke-Dahl S, Möller CG, Kelly PM, et al. Localization of USH2B to 5q14.3 - q21.3. Molecular Biology of Hearing and Deafness Conference, Bethesda, MD, USA, 1998:abstract 88.

17 Dib C, Faure S, Fizames C, et al. A comprehensive genetic map of the human genome based on 5,264 microsatellites. Nature 1996;380:152-4.

18 Bateman JB, Riedner ED, Levin LS, Maumenee IH. Heterogeneity of retinal degeneration and hearing impairment syndromes. Am f Ophthalmol 1980;90:755-67.

19 Innis JW, Sieving PA, McMillan P, Weatherly RA Apparently new syndrome of sensorineural hearing loss, retinal pigment epithelium lesions and discolored teeth. Am f Med Genet 1998;75:13-17.

20 Liu XZ, Hope C, Walsh J, et al. Mutations in the myosin VIIA gene cause a wide phenotypic spectrum, including atypical Usher syndrome. Am ₹ Hum Genet 1998;63:90912 .

21 Pereira FA, Qiu T, Tsai MJ, Tsai SY. Chicken ovalbumin upstream promoter transcription factor (COUP-TF): expression during mouse embryogenesis. F Steroid Biochem Mol Biol 1995;53:503-8.

22 Qiu Y, Krishnan V, Zeng Z, et al. Isolation, characterization, and chromosomal localization of mouse and human COUP TF-I and II genes. Genomics 1995;29:240-6. 